\title{
Brasil nuclear: dos interpretaciones opuestas sobre la orientación de su programa atómico
}

Nuclear Brazil: Two Opposed Interpretations about the Orientation of its Atomic Program

\author{
JUAN FRANCISCO MORALES GIRALDO
}

Instituto de Estudios Políticos Andinos (IEPA), Perú

RESUMEN: Al analizar el programa nuclear de Brasil desde dos enfoques teóricos distintos, su orientación y propósitos presentan también importantes diferencias. Las perspectivas más optimistas, de corte liberal, enfatizan que la política exterior de un país debería juzgarse desde aspectos favorables a los valores democráticos, el respeto por las normas internacionales y la autoevaluación de la legitimidad frente al escrutinio de la opinión pública. Pero al contrario de enfoques que se sostienen en argumentos normativos, una perspectiva basada en una política de poder incidiría en aspectos menos idealistas: la reconfiguración de la jerarquía del poder internacional y las aspiraciones de una potencia global emergente como motivaciones subyacentes para los desarrollos técnicos del programa atómico. Este trabajo sopesa la relativa utilidad de ambas perspectivas, enfocándose en situaciones, desarrollos y discursos del pasado reciente que permiten inferir la orientación política el programa nuclear del Brasil.

El estudio procede contrastando las evidencias recogidas con elementos teóricos asociados a cada perspectiva. En una primera parte, se compara la política exterior del Brasil respecto al régimen de no proliferación con lo que las tesis liberales plantean acerca de la conducta estatal. En ese sentido, tendrían especial importancia explicativa el peso normativo de las instituciones internacionales, la democracia interna y la legitimidad que otorga el apego a estos principios. En el segundo apartado se adopta una perspectiva opuesta: las tesis liberales respecto a la problemática del programa nuclear del Brasil son vistas de manera crítica según una interpretación de su orientación basada en una política revisionista y pragmática en consonancia con objetivos mayores de estatus internacional y relaciones de poder con las grandes potencias. La evidencia más importante, la ambigüedad de un programa nuclear pacífico con fines militares, sugeriría que es la segunda perspectiva la que posee mayor capacidad explicativa de los propósitos del Brasil en esta materia.

Las conclusiones del estudio se enfocan en tres distinciones esenciales entre ambos enfoques que responden a las interrogantes planteadas: la ausencia de un programa de armas nucleares en contraste con la militarización de la tecnología nuclear y sus aplicaciones; el apego a la institucionalidad internacional en contraste con el pragmatismo y la selectividad en el cumplimiento de ciertos principios; y, finalmente, las fronteras conceptuales poco claras que quedan expuestas por el programa nuclear del Brasil.

PALABRAS Clave: Brasil, Proliferación nuclear, Liberalismo político, Realismo, Tecnologías de doble uso. 
ABSTRACT: The analysis of the Brazilian nuclear program from different theoretical approaches shed light on differences regarding its orientation and purposes. Optimistic views, from a liberal standpoint, emphasize the idea that States' foreign policy should be asses in a favorable fashion according to certain aspects such as democratic values, compliance with international norms and legitimacy from public opinion. But in opposition to perspectives based on normative arguments, a power politics perspective emphasizes least idealist aspects: the reconfiguration of international power hierarchy and the aspirations of an emergent global power as subjacent motivations for the technical developments of the atomic program. This work ponders the relative utility of both perspectives focusing on situations, developments and discourses from recent past that make possible to infer the political orientation of Brazilian nuclear program.

The study proceeds by contrasting the evidence available with theoretical elements from each perspective. First, Brazilian foreign policy regarding the Nonproliferation Regime is compared with assumptions from Liberal thesis about State conduct. In that sense, aspects such as the weight-normative international institutions, domestic democracy and legitimacy provided by these principles, would have an important explanatory importance. In the second part, an opposed view is adopted: the liberal thesis applied to the problematic of Brazilian nuclear program are seen critically from the standpoint of an interpretation about its orientation based on a revisionist policy and pragmatism related to major objectives of international status and power relations with Great Powers. An important evidence in that sense is the ambiguity of the nuclear program and its military purposes, which suggest that the second perspective offers a better explanation of Brazilian objectives in this area.

Conclusions are focused on three distinctions between both perspectives that give answers to the problems of the study: the absence of a nuclear program in contrast with the militarization of the nuclear technology and its applications; the acceptance of international institutions in contrast with the pragmatism and selectiveness of the compliance of certain principles; and finally, the unclear conceptual frontiers exposed by the Brazilian nuclear program.

KEYWORDS: Brazil, Nuclear proliferation, Political liberalism, Realism, Dual-use technologies.

Recibido: 14 de agosto de 2020. Aceptado: 27 de octubre de 2020.

Revista de Estudios en Seguridad Internacional, Vol. 6, No. 2, (2020), pp. 81-100. http://www.seguridadinternacional.es/revista/

ISSN: 2444-6157. DOI: http://dx.doi.org/10.18847/1.12.5 


\section{INTRODUCCIÓN: EL VIRAJE POLÍTICO Y EL PAPEL DE LA SEGURIDAD Y LA DEFENSA}

Brasil ha experimentado una transformación significativa en su sistema político desde mayo de 2016. Las derrotas electorales del Partido de los Trabajadores (PT), el impeachment y posterior destitución de la entonces presidenta Dilma Rousseff (20112016), y el encarcelamiento del expresidente Lula da Silva (2003-2010), dieron paso a una transición desgastante para el país y las fuerzas políticas que lo configuraron, desde la nueva izquierda progresista del siglo XXI hacia la radicalización conservadora de sus protagonistas más recientes. El rechazo popular que suscitó la presidencia interina del constitucionalista conservador Michel Temer (2016-2018), desacreditó aún más las estructuras intermedias que sirvieron de soporte para el progresismo de los últimos quince años, y desalentó la confianza en un sistema político deteriorado, incapaz de contener los efectos de la crisis económica: el PBI del país se contrajo en un 3.8\% en 2015 y $3.6 \%$ en 2016 (Instituto Brasileño de Geografía y Estadística). En el terreno electoral, las mayorías optaron entonces por un cambio de rumbo con la elección de Jair Bolsonaro (2019), un ex capitán del Ejército de tendencias reaccionarias en el espectro de la extrema derecha, como presidente de la República. El país se encontraba ya sobre la ola del populismo global en los albores de la tercera década. El potencial de estos cambios para trastocar elementos definitorios del régimen político (las ideologías, valores y creencias prevalecientes, las reglas del juego político y las estructuras de autoridad) sellaron el fin del ciclo progresista de la primera década, referido no sólo a la caída de los gobiernos que lo personificaron, sino a una forma diferente de ejercer y comprender el poder (Schavelzon, 2017).

Los cambios en el régimen político y el carácter del nuevo gobierno, generaron, como es de esperarse, cambios en la conducción del país en general y en su política exterior en particular. Desde 2016, el país inició un rápido repliegue de las principales instituciones regionales que habían sido promovidas en la corta era del regionalismo post-hegemónico (Tussie \& Riggirozzi, 2012) o post-liberal (Da Motta y Rios, 2007; Sanahuja, 2014). El gobierno de Lula da Silva, a través de las gestiones de su ministro de exteriores, Celso Amorim, un destacado académico y diplomático, concibieron una gran estrategia de política exterior ${ }^{1}$ en la cual la creación y promoción de un sistema de gobernanza regional fuera un factor fundamental para la proyección internacional del país como una gran potencia en ascenso. En palabras del exministro Amorim, Brasil se reconocía como un "firme creyente" en el multilateralismo, un concepto que consideraba normativo (Amorim, 2010: 218; 2013). Para los gobiernos de la nueva izquierda, esta nueva institucionalidad formaba parte de un gran esfuerzo contra-hegemónico que garantizaría a los países de Sudamérica la salvaguarda de su independencia y autonomía (Russell \& Tokatlian, 2003; Zópel, 2018). Para Brasil y sus objetivos a mediano plazo, las nuevas organizaciones internacionales, Unasur y sus diversos órganos sectoriales, que por primera vez se distinguían por un alcance realmente regional, conformaban una red de relaciones de distinto orden a través de las cuales ejercer una influencia política mayor en diversos asuntos de interés común, siendo éste el fundamento para un liderazgo efectivo (Morales Giraldo, 2018). Al mismo tiempo, en un contexto económico global favorable, en el transcurso de la década de 2010 el país se convirtió en la sexta mayor economía del mundo (siendo la octava actualmente, según el Fondo Monetario Internacional [FMI], 2019) ${ }^{2}$, a la vez que se atendían aspectos de la seguridad nacional frente a nuevas

\footnotetext{
${ }^{1}$ Sobre el concepto de "gran estrategia", ver Feaver (2009).

${ }^{2}$ Así lo señalaba, por ejemplo, una nota de BBC Mundo del 26 de diciembre de 2011 titulada "Brasil: 'la sexta economía del planeta"”. Sin embargo, para 2017, el portal informativo de la CIA, World Factbook
} 
amenazas emergentes aunque inciertas (Ministerio de Defensa del Brasil, 2008). En resumen, la gran estrategia del anterior gobierno progresista reconocía los elementos de poder económico, militar y político como piezas fundamentales de su política exterior. Sin embargo, fueron los posteriores gobiernos conservadores los que desestimaron gran parte de estos avances: el repliegue institucional, que llevó al país a retirarse o restar apoyo a las principales organizaciones de América del Sur (principalmente, Unasur y su Consejo de Defensa como marcos de coordinación política), supuso cortar vínculos que configuraban gran parte de sus capacidades de poder relacional, quedando únicamente el poder estructural, en su dimensión agregada, como sostén de su proyección internacional ${ }^{3}$. Esto es ilustrativo de la tesis de Schavelzon, aplicada a la política exterior: el desconocimiento de la importancia de los vínculos institucionales para el ejercicio del poder político (un componente del poder relacional), reafirmando a través de las acciones la sola importancia de las capacidades y recursos individuales (poder estructural, hoy disminuido), es ciertamente una nueva forma diferente de ejercer y comprender el poder.

A pesar del drástico cambio de rumbo, quedaron importantes elementos de continuidad. Los principales programas de adquisición de armas, iniciados durante el gobierno de Lula da Silva, se mantuvieron apenas con algunos ajustes: el Programa Gripen-E/F (Projeto FX-2) para la fabricación de los nuevos cazas de diseño sueco de la FAB; el Programa Clase Tamandaré de la Marinha (Proyecto Construcción del Núcleo de Poder Naval), a cargo del consorcio Águas Azuis liderado por la alemana Thyssenkrupp y la brasileña Embraer Defence \& Security, para la construcción de cuatro fragatas multipropósito; El Programa de Desarrollo de Submarinos (PROSUB), que contempla el diseño y construcción de un submarino de propulsión nuclear (denominación SN-BR) y cuatro submarinos diésel-eléctricos basados en la Clase Scorpène; el programa de desarrollo y fabricación del avión de transporte medio Embraer C-390 Millennium; y el programa ASTROS 2020, dentro del cual se desarrolla el misil táctico de crucero MTC-300 del Exército Brasileiro. La continuación de estos proyectos en el marco de políticas para la seguridad y defensa responde a dos factores, uno estructural y otro contingente: en Brasil, la modernización de las Fuerzas Armadas fue siempre una cuestión de Estado, un conjunto de medios disuasivos frente a posibles situaciones de injerencia, intervención o sometimiento de poderes externos, pero también necesarios para la ocupación efectiva de áreas de interés, la presencia del Estado en el territorio nacional y el mantenimiento del orden interno (Ministerio de Defensa del Brasil, 2012: 24) ${ }^{4}$. Por otro lado, el gobierno de Jair Bolsonaro ha significado el retorno de (ex) miembros de las Fuerzas Armadas a posiciones de dirección en la administración del Estado por primera vez desde 1985 (ejemplos notables: el actual vicepresidente, Hamilton Mourão, es un General en retiro, y el jefe de la Oficina Presidencial, Walter Souza Braga, un General en servicio activo). Por estas razones, aunque la política exterior experimentara un importante cambio de prioridades, la política de seguridad y defensa ha mantenido sus objetivos y metas a mediano plazo sin alteraciones, especialmente los relacionados con la industria y el desarrollo de tecnologías militares.

Uno de los aspectos destacables entre estos rasgos de continuidad es el renovado apoyo al desarrollo y continuidad del programa nuclear y la dirección militar. El anterior titular

\footnotetext{
situaba a Brasil en el puesto $8^{\circ}$ de las mayores economías ordenadas según el PBI ajustado a la paridad de poder adquisitivo, según estimaciones de ese año.

${ }^{3}$ Sobre los tipos de poder mencionados, ver Habeeb (2000: 83).

${ }^{4}$ Así también, el equipamiento de las Fuerzas Armadas, a través de un adecuado presupuesto, se establece como una meta prioritaria en el Plano Brasil 2022 de la Secretaria de Assuntos Estratégicos - Presidência da República, y entre las directrices de la Estrategia Nacional de Defensa (Directriz 21) (2008: 17-18).
} 
de la Dirección General de Desarrollo Nuclear y Tecnológico de la Armada (DGDNTM), principal organismo dedicado a este objetivo, el Almirante Bento Costa Lima Leite, ocupaba hasta este año el Ministerio de Minas y Energía. En su antiguo puesto, el Almirante Costa Lima Leite tenía responsabilidad directa sobre todas las unidades científicas de la Marinha, incluyendo el Programa de Desarrollo de Submarinos (PROSUB) y el Programa Nuclear da Marinha (PNM), además de ocupar la Presidencia de Consejo de la empresa estatal Amazônia Azul Tecnologias de Defesa (Amazul) (Dantas, 2017; Mazui, 2018). Su presencia en el Gabinete de Ministros acerca las políticas de la Presidencia al conocimiento y la experiencia generados en las principales instancias técnicas y de gestión del programa nuclear. Así también, los principales programas y proyectos del programa siguen en vigencia, y los principios de la política general del país en materia de seguridad y defensa - el sector al cual responden los principales intereses, se mantienen. Con un gobierno conformado por notables figuras del ámbito militar, el cambio de gobierno ha supuesto un cambio de énfasis para el programa atómico, tal como sostiene el analista Leonardo Bandarra (2019). Pero existen espacios de una cierta indeterminación que parecen también perdurar pese al nuevo rumbo político: ¿hacia qué objetivos se dirige, finalmente, el programa nuclear brasileño? La ambigüedad fundamental que entraña un programa nuclear pacífico destinado a usos militares gestionado y dirigido por militares - requiere ser reinterpretada a la luz de los principios elementales detrás de su concepción como instrumento de estrategia política en el más amplio campo de las relaciones internacionales, principalmente debido a su potencial efecto disruptor sobre los actuales equilibrios que sostienen la llamada Revolución Nuclear (Jervis, 1986). Estas re-interpretaciones pueden darse desde dos perspectivas opuestas, aunque no del todo contradictorias. Una perspectiva optimista, que valora los efectos políticos del programa nuclear en su aspecto más favorable para la estabilidad internacional, destacando la política exterior del Brasil desde la posición de un actor internacional responsable y reafirmando el papel prescriptivo de la democracia, los acuerdos y la opinión pública como categorías normativas sobre el comportamiento estatal. Del otro lado se ubica una perspectiva de corte realista, que acentúa con un sentido práctico las dinámicas que sostienen la jerarquía del poder, el revisionismo como tipo de política exterior, y la naturaleza militar del propio programa, como categorías de análisis.

Hacia la primera década de los años 2000, quienes insistían con optimismo en entender los propósitos políticos del programa nuclear brasileño como un proyecto exclusivamente pacífico, incluso no militar, interpretaban su desarrollo como parte del progreso natural de un líder internacional emergente, democrático, responsable hacia la institucionalidad internacional y el mantenimiento de la paz en sus relaciones con los demás miembros de la comunidad internacional. Quienes, por otra parte, llamaban la atención sobre la ambigüedad de su política nuclear y la orientación de sus desarrollos tecnológicos, la consideraban funcional a una política revisionista orientada a reconfigurar el equilibrio de poderes en un orden internacional desigual. Ambas perspectivas difieren en cuanto a cómo valorar la ambigüedad de un programa nuclear pacífico con fines militares, los usos de la energía nuclear y sus implicaciones para la seguridad internacional. En el primer caso, la presencia o ausencia de un programa para el desarrollo de armas nucleares sería determinante a la hora de identificar los posibles riesgos para la estabilidad internacional. Desde una perspectiva opuesta, en cambio, el amplio espectro de potenciales usos militares de la energía nuclear, aún sin un programa de armas, se entendía como respuesta a un entorno de múltiples amenazas emergentes, reales o no, que hacían necesarias acciones de equilibrio para la reafirmación de la propia autonomía. Gran parte de estas ideas contrapuestas adquirieron fuerza durante la etapa del gobierno del PT bajo el liderazgo de Lula da Silva, periodo en el cual el programa nuclear del Brasil inició una 
nueva etapa de avances técnicos con mayor respaldo político. Los objetivos políticos del programa fueron establecidos durante este periodo, coincidiendo con una mayor proyección del Brasil como actor internacional de primer orden. El análisis a continuación sopesa la relativa utilidad de ambas perspectivas, enfocándose en situaciones, desarrollos y discursos del pasado reciente que permiten inferir hacia dónde se dirige (políticamente) el programa nuclear del Brasil.

\section{DEMOCRACIA, INSTITUCIONES Y PRESTIGIO}

Las perspectivas optimistas comparten la convicción de que los Estados guían su política exterior según preceptos ligados a la superioridad moral de la democracia, el respeto de las normas del derecho internacional público y la autoevaluación de la legitimidad de sus acciones - en un sentido ético - frente al escrutinio de la opinión pública. En la teoría, esta idea común, compartida por políticos y analistas, fue integrada en los planteamientos del liberalismo político: las democracias liberales modernas tienden a privilegiar los patrones de cooperación en sus relaciones exteriores a través de mecanismos institucionales sobre los cuales proyectan un mayor compromiso que los Estados no democráticos (ver Jackson \& Sørensen, 2007: 135; Keohane, 1993: 28-29; 2009: 57-70; Martin, 2003: 92). La superioridad moral de la democracia, la institucionalidad de las normas del derecho internacional y el tribunal de la opinión pública, se convierten entonces en factores que promoverían la paz y la cooperación al establecer los límites de lo permisible para la acción de los Estados (ver Holsti, 1992). Desde esta perspectiva, las implicaciones del programa nuclear del Brasil para la seguridad internacional fueron evaluadas respecto a las características de su política interna (la democracia), el carácter de su política exterior (un líder emergente) y la auto-evaluación de su papel como actor internacional a partir de estos aspectos. La transición democrática iniciada a mediados de la década de 1980 es considerada como el precedente más importante en la reorientación del programa atómico hacia fines pacíficos con el abandono de las tareas de investigación y desarrollo de armas nucleares (Flemes, 2006: 25; Mauger, 2012; Patti, 2010: 196; Santos Vieira de Jesús, 2011: 119). Pero lejos de basarse sólo en planteamientos teóricos o preconceptos comunes, para las perspectivas optimistas el carácter pacífico del programa nuclear del Brasil sería una verdad histórica, un hecho destacable, demostrable en el sentido de que la democracia, las instituciones y el prestigio del país habrían operado, en efecto, como condicionantes en el diseño de sus propósitos.

En el Cono Sur, la transición democrática había favorecido el surgimiento de un patrón general de relaciones con mayor énfasis en las dinámicas de cooperación que de conflicto (Kahhat, 2007). El cambio de régimen en Brasil desde 1985, como en otros países de la subregión en ese periodo, generó expectativas sobre un cambio de orientación en su política nuclear hacia el desarrollo de aplicaciones no militares de la energía atómica y de tecnologías asociadas a su uso. La desaparición de las doctrinas de seguridad tradicionales como ejes de la política exterior, y el inicio de la cooperación nuclear bilateral entre Argentina y Brasil, refrendada a través de mecanismos como las Declaraciones Conjuntas sobre Política Nuclear de 1985 (Iguazú), 1986, 1987 (Viedma), 1988 (Iperó) y 1990, y el Acuerdo de Guadalajara para el Uso Exclusivamente Pacífico de la Energía Nuclear (llamado también Acuerdo Bilateral) de 1991 (Bompadre, 2000: 56-57; Mauger, 2012), ayudaron a desestimar la necesidad de parte de ambos países por desarrollar programas nucleares militares que habrían de garantizar el equilibrio estratégico que había obsesionado a los regímenes militares. Precisamente con el Acuerdo de 1991 se creó la Agencia Brasileño-Argentina de Control y Contabilidad de Materiales Nucleares (ABACC), organización específicamente destinada a gestionar y aplicar las 
salvaguardias del Sistema Común para Contabilidad y Control de Materiales Nucleares. Con esto, la política nuclear del Brasil quedó firmemente establecida dentro de un marco de normas y compromisos internacionales. En otro plano de circunstancias, en cuanto a política interna, la renuncia del Brasil a las armas nucleares había quedado prescrita en la Constitución de 1988, cuyo Artículo 21 prohibió desde entonces la utilización de la energía nuclear para fines que no sean exclusivamente pacíficos (Waiman, 2010: 499). Finalmente, el abandono de los planes de desarrollo de armas de destrucción masiva podía considerarse consecuencia del propio cambio de régimen y la nueva orientación de su política exterior.

Durante el periodo posterior de consolidación democrática desde la década de 1990, Brasil se mostró a sí mismo como un país comprometido con las normas internacionales sobre la utilización pacífica de la energía nuclear. Su adhesión al Tratado de No Proliferación de Armas Nucleares (TNP) en 1997 supondría un punto de quiebre hacia la prescripción de armas nucleares como un objetivo central en su política de seguridad durante los gobiernos de Fernando Henrique Cardoso y Luiz Inácio Lula da Silva, agendada en un marco de compromiso y responsabilidad hacia la institucionalidad del régimen de no proliferación (Mauger, 2012; Patti, 2010: 181-182, 196; Santos Vieira de Jesús, 2011: 119). Considérese que, aunque el TNP prescribe el desarrollo de armas nucleares, rescata, sin embargo, el derecho inalienable de todos los Estados a desarrollar la energía nuclear para fines pacíficos (Artículo IV). En ese sentido, la transición democrática en lo concerniente a la política interna, así como el propio régimen de no proliferación nuclear, sus principios, normas y procedimientos, habrían ejercido importantes efectos normativos sobre la orientación y los propósitos generales de la política nuclear brasileña. La literatura recoge evidencias de que los Estados no democráticos serían más propensos a desconocer sus compromisos con el TNP (Miller \& Sagan, 2009: 11). En este caso, ambos hechos reforzaron la convicción de que la democracia y las instituciones internacionales podían ser decisivas para el replanteamiento de un programa nuclear con fines estrictamente pacíficos.

La conjunción histórica de estas condiciones — democratización, institucionalidad de los acuerdos internacionales, y no proliferación, al menos en la práctica- dio pie a aproximaciones más optimistas respecto al uso militar de la energía nuclear como parte de los cambios que experimentaba el país. Sin embargo, ya en el terreno del análisis, hechos históricos como la instauración de un nuevo gobierno democrático y el progresivo acercamiento al régimen de no proliferación como marco legítimo para una política nuclear reconocida por la comunidad internacional, presentan algunas limitaciones como factores explicativos de un supuesto cambio de orientaciones y propósitos. En primer lugar, porque argumentos similares basados en las tesis liberales que otorgan importancia a la democracia y las instituciones como elementos de dirección de una política exterior, no admiten continuidades entre sistemas políticos opuestos - la transición de una dictadura militar a una democracia en construcción-, con lo cual los cambios deberían poder demostrarse, principalmente, respecto al desarrollo de la política nuclear brasileña en sus aspectos políticos: por ejemplo, el papel de los militares, la distribución de responsabilidades entre las nuevas autoridades e instancias de gobierno, pero, sobre todo, respecto a sus fines generales - un punto será analizado en el siguiente apartado. En segundo lugar, la importancia de las instituciones internacionales como factores condicionantes de las relaciones internacionales depende de la presencia de intereses mutuos entre las partes involucradas y de efectos sustanciales de las variaciones institucionales sobre el comportamiento de los actores políticos —en este caso, un 
Estado- (Keohane, 1993: 15-16). La evaluación de ambos puntos nos lleva a adoptar una posición crítica frente a los argumentos vistos hasta ahora.

En el marco del acuerdo, el reconocimiento de objetivos comunes no fue un problema entre los Estados parte en el TNP. Los tres pilares del tratado: el desarme de los arsenales nucleares ya existentes, la no proliferación y el derecho al uso pacífico de la tecnología nuclear $^{5}$, eran metas a largo plazo ampliamente aceptadas, al menos nominalmente. Sin embargo, dado que su cumplimiento estuvo siempre condicionado, sí existían objeciones a su no-cumplimiento, sobre lo cual las potencias emergentes habían sido muy críticas. Esta insatisfacción ha sido también un aspecto de continuidad en la política nuclear del Brasil, desde los tiempos del gobierno militar a la nueva democracia, aunque sus implicancias suelen ser obviadas por los análisis más optimistas, convencidos de su compromiso incondicional con la normatividad del tratado. En mayo de 2010, y a propósito de la crisis nuclear iraní en la que Brasil intervino como mediador, sin resultados, el entonces presidente Lula da Silva afirmó que eran las armas nucleares, y no los acuerdos con Irán, lo que hacían al mundo más peligroso (La Nación, 2010). En otra ocasión, durante su participación en la Conferencia de Estados Parte en el TNP, el ministro Celso Amorim expresó la convicción de su gobierno de que los arsenales nucleares tuvieran alguna utilidad, exigió su desmantelamiento, y defendió el argumento de que "mientras algunos Estados posean esos armamentos, otros tendrán la tentación de adquirirlos o desarrollarlos" (Organización de las Naciones Unidas [ONU], 2010). Un año después, en septiembre de 2011, la presidenta Rousseff abogó ante la Asamblea General de Naciones Unidas por "la eliminación completa e irreversible" de todos los arsenales nucleares (El Universal, 2011). Dado lo explícito de estas declaraciones, es evidente que la insatisfacción con el no cumplimiento de los mandatos del régimen de no proliferación nuclear no contribuía precisamente a la existencia de intereses compartidos entre los Estados que poseen armas nucleares y los que no, lo que sin duda debilita la institucionalidad del tratado.

Tradicionalmente, Brasil ha considerado los arsenales nucleares como un privilegio de aquellos que sostienen y promueven una concepción asimétrica del mundo (El Universal, 2011), por lo que no resulta extraño comprobar que su resistencia a una ampliación de los mecanismos de fiscalización, control y salvaguardias del Organismo Internacional de Energía Atómica (OIEA) y a las restricciones del TNP, sea justificada por consideraciones de interés nacional vinculadas a una posible vulneración de sus potestades soberanas (La Voz del Mundo, 2010). Según su política nacional de seguridad y defensa, el sector nuclear posee un alto valor estratégico cuyo imperativo es mantener abierto el camino hacia un desarrollo nuclear autónomo (Ministerio de Defensa del Brasil [MDB], 2008: 34). Uno de los aspectos que más llaman la atención en el documento es constatar que el desarrollo nuclear de Brasil, en cuanto a cómo se conciben sus propósitos, no está sujeto de manera incondicional a la normatividad del TNP en particular y del régimen de no proliferación nuclear en general: su renuncia a la obtención de armas nucleares fue hecha bajo ciertas premisas, la más importante de las cuales era el progresivo desarme de los arsenales ya existentes (MDB, 2008: 33), uno de los pilares del régimen de no proliferación, aunque, también, uno de sus mandatos de menor

\footnotetext{
${ }^{5}$ Para motivar a los Estados a unirse al Tratado de No Proliferación, sus principales proponentes, Estados Unidos y la Unión Soviética, llegaron al acuerdo de que las obligaciones destinadas a detener la proliferación de armas atómicas debían ir acompañadas de obligaciones que facilitaran el acceso a los usos pacíficos de la energía nuclear, y de progresos hacia un eventual desarme. Estas obligaciones fueron establecidas en los Artículos II y III del tratado (referentes a la no proliferación), Artículo IV (el uso pacífico de la energía nuclear), y Artículo VI (desarme). Ver Dalton et al. (2017), Carlson (2019: 98-99).
} 
cumplimiento y efectividad (Windt, 2017; para una aproximación más optimista al asunto, véase Carlson, 2019).

Finalmente, al margen de toda controversia y pese a sus relativas limitaciones, la transición a un sistema democrática y el apego a la institucionalidad del Tratado de NoProliferación, serían también fuente de legitimidad y prestigio ante la opinión pública, importante para establecerse como un actor a tener en cuenta en áreas sensibles de los asuntos internacionales, y como un firme candidato a ocupar un asiento permanente en el Consejo de Seguridad, una vieja aspiración del Brasil (Flemes, 2006: 29-30; Mauger, 2012; Santos Vieira de Jesús, 2011: 119). Un elemento como este, no obstante, induce el análisis a una aparente dualidad moral que antepondría la orientación pacífica de programas nucleares como el de Brasil a los peligros asociados a la nuclearización de los llamados Estados paria, indeseables y no democráticos, sin atender a la ambigüedad del primero en cuanto a los usos claramente militares de la tecnología nuclear. El resultado sería una apreciación valorativa sobre la renuncia del Brasil a la obtención de armas nucleares que no tiene en cuenta el pragmatismo que ha caracterizado su política nuclear en las últimas décadas. A decir del connotado periodista Andrés Oppenheimer (2009), "lo más probable es que Brasil desarrolle un programa nuclear con fines pacíficos, como lo afirma la postura oficial, porque quiere seguir siendo un buen ciudadano global".

Los análisis optimistas tienden a atribuir al programa nuclear brasileño fines pacíficos sin tener en consideración la ambigüedad de la utilización militar de las tecnologías asociadas. Sin embargo, sus rasgos normativos presentan dificultades para explicar un amplio conjunto de evidencias acerca de cómo el cálculo político-estratégico, y no el tipo de régimen, el apego a las normas o las convicciones éticas, serían determinantes las decisiones del Brasil sobre la orientación general de sus políticas en esta cuestión. Las perspectivas de poder enfocadas en las dinámicas competitivas que subyacen al orden internacional, y cómo los Estados revisionistas se adaptan a ellas, presentan una alternativa de análisis a estas interpretaciones.

\section{UNA REINTERPRETACIÓN DE PROPÓSITOS: EL REVISIONISMO DE LA POLÍTICA EXTERIOR}

Al contrario de posiciones que otorgan una importancia considerable a aspectos normativos de la política internacional, el amplio - aunque no del todo claro- panorama de aplicaciones militares de la energía nuclear que ha abierto el Brasil dan sustento a explicaciones que inciden en aspectos menos idealistas: la reconfiguración de la jerarquía del poder internacional y las aspiraciones estratégicas de una gran potencia emergente como motivaciones subyacentes a los alcances técnicos del programa atómico. Este revisionismo - es decir, la insatisfacción con las condiciones generales existentes referidas a territorio, jerarquía, poder y valores - contrasta con la arraigada opinión acerca de la naturaleza pacífica del programa nuclear y la creencia común de que las relaciones de poder serían, necesariamente, un antecedente a la violencia interestatal, y por esa razón deberían evitarse sus extremos. En la literatura sobre la problemática de la proliferación nuclear, esta aproximación de corte realista suele ser erróneamente reducida al estudio exclusivo de las amenazas militares externas (Mauger, 2012; Sagan, 19961997: 57-63), una exageración de sus presupuestos básicos. En la teoría, estas ideas, fueron integradas en los planteamientos del realismo político.

La tesis que se desarrolla a continuación parte del presupuesto de que las asimetrías en las condiciones materiales de los Estados, sensibles a su situación, los empuja a adoptar ciertos patrones de política exterior para la salvaguarda de intereses fundamentales como son la autonomía política, la seguridad y el bienestar (Grieco, 1989: 498-499; 
Mearsheimer, 2001; Waltz, 1979: 91, 102-104, 126, 131). En ese sentido, es importante destacar la presión que ejerce un sistema internacional jerarquizado, asimétrico y polarizado sobre la política exterior de aquellos Estados que ven en estas condiciones un peligro potencial (real o no) para su independencia e integridad. No se trata, en todo caso, de una hipotética lucha por la supervivencia (a decir de Morgenthau, 1986), sino de reconocer el hecho de que, para una potencia revisionista, las capacidades militares, incluyendo aquellas tecnologías con el potencial de elevar su poderío, constituyen el más importante factor material que contribuye a conformar el poder de una nación, esto es, su capacidad para influir ventajosamente en sus relaciones y su contexto.

Desde esta perspectiva, las aplicaciones militares de la energía nuclear del Brasil serían consideradas funcionales a una política exterior orientada a la reconfiguración de la jerarquía del sistema internacional, un propósito esencialmente político. Esto no supone necesariamente la producción de armas nucleares, ni agota las posibilidades abiertas por las tecnologías de doble uso sobre intereses económicos (especialmente ligados a aplicaciones comerciales de ciertos desarrollos técnicos y el impulso a la competitividad de la industria local; véase Lomax, 2001). La relación, por una parte, entre el poderío nuclear, a través del dominio de tecnologías apropiadas, y la fuerza militar como elemento constitutivo del poder estatal por otro lado, abren un panorama de situaciones que transcienden la problemática de la proliferación de la bomba atómica como único aspecto a tener en cuenta. La adaptación de tecnologías de doble uso para la propulsión naval de submarinos de ataque, cruceros y portaviones, deja en obsolescencia la idea de que las armas de destrucción masiva son la única vía técnica en el ámbito militar, lo que de otra manera habría llevado a creer, equivocadamente, que el programa nuclear brasileño, sin la bomba atómica, estaba orientado a fines civiles y no militares.

La orientación hacia tecnologías nucleares de doble uso para aplicaciones tanto civiles como militares confluyeron históricamente en un interés común: la autonomía técnica en el enriquecimiento de uranio. El Programa Autônomo de Tecnologia Nuclear (PATN), creado en 1979 en paralelo al programa oficial ${ }^{6}$, fue concebido como respuesta a las crecientes restricciones a la transferencia de tecnologías clave y combustible nuclear por parte de Estados Unidos y el Grupo de Abastecedores Nucleares, fundado en 1975 (Barletta, 1997: 13-14; MDB, 2012: 68; Nedal, s.d.; Patti, s.d.). La presión ejercida por potencias externas impulsó a civiles y militares a colaborar en un programa que permitiera, por un lado, lograr la autonomía técnica de los suministros energéticos provenientes del restrictivo mercado exterior (y otras aplicaciones civiles, económicas y comerciales), así como plantear una respuesta apropiada a partir de consideraciones estratégico-militares al entonces naciente programa nuclear argentino, (Barletta, 1997: 15). Entre 1985 y 1990, el mismo periodo de transición democrática, las pugnas burocráticas entre el programa oficial (bajo coordinación del Ministerio de Energía y Minas y Nuclebrás Equipamentos Pesado S.A. - NUCLEP) y el PATN (bajo coordinación

\footnotetext{
${ }^{6}$ El programa oficial fue creado en 1975 en cooperación con la República Federal Alemana durante la presidencia de Ernesto Geisel. El convenio, bajo los controles y la supervisión del OIEA, incluía la construcción de una planta piloto de enriquecimiento de uranio por procesos aerodinámicos (técnica Becker Jet-Nozzle) y otra de reprocesamiento del combustible utilizado, la exploración y la extracción de uranio y la construcción de plantas de energía nuclear. La oposición de los Estados Unidos (que veía al gobierno militar de Brasilia como un posible proliferador) consiguió que el gobierno alemán excluyera del acuerdo el método de enriquecimiento por ultracentrifugación. A la postre, el método transferido (Becker JetNozzle) resultaría tecnológica y económicamente impracticable, lo que supondría el fracaso del programa oficial. Ante las restricciones internacionales, el PATN estaría destinado fundamentalmente al desarrollo autónomo de una tecnología adecuada para el enriquecimiento de uranio (Barletta, 1997: 4; Nadal, 1991: 554; Nedal, s.d.; Patti, s.d.).
} 
de la Comissão Nacional de Energia Nuclear) terminaron por favorecer a este último, sobre todo tras su éxito en el manejo de los procesos de enriquecimiento de uranio en 1987 (Barletta, 1997: 22-23). El PATN logró responder exitosamente a las necesidades civiles y militares para el que fue concebido, y pese a que la crisis económica retrasó el programa durante muchos años, fue reimpulsado en la primera década de los años 2000. El resultado de estos desarrollos técnicos fue un programa cuya dimensión militar heredaría el importante papel de servir como pieza estratégica en la política de seguridad y defensa del país ${ }^{7}$.

La transición democrática no supuso un cambio de rumbo para el programa nuclear brasileño. En cierto modo, la percepción compartida por civiles y militares sobre los efectos negativos de las restricciones del régimen internacional de no proliferación (especialmente de parte de los Estados Unidos), consolidó el desarrollo nuclear autónomo como una política de Estado desde etapas tempranas de su concepción en la década de 1950, resaltando, además, que desde entonces la autonomía relativa de las Fuerzas Armadas, principales instituciones gestoras del programa atómico, tuvo un papel importante en su continuidad (sobre la evolución de una política nuclear independiente, ver Grabendorff, 1987: 531-534). Con la instauración de la democracia en Argentina en 1983, la desaparición de las hipótesis de conflicto mutuo, y el advenimiento de la integración económica y la cooperación política institucional a través del nuevo Mercosur, el propósito central y perdurable de la dimensión militar del programa nuclear brasileño ya no era responder ante una amenaza militar externa directa, sino servir como un instrumento estratégico capaz de brindar al país los vectores de poder adecuados a una potencia global emergente (sobre la convergencia nuclear entre ambos países con el restablecimiento de la democracia, ver Bompadre, 2000: 56-58). La estrecha relación entre seguridad y desarrollo tecnológico industrial, que incluye a la energía nuclear, ha sido reconocida oficialmente en la Estratégia Nacional de Defesa como un eje esencial para establecer las condiciones y requisitos materiales del poder militar nacional (Barletta, 1997: 17; MDB, 2008: 33).

Precisamente, el impulso a la iniciativa para la construcción de submarinos de propulsión nuclear, el más importante componente del programa nuclear en conjunto, es un ejemplo de la continuidad de estas políticas. Tras la firma de acuerdos de cooperación técnico-militar entre Brasil y Francia en 2008 (en los que la transferencia de tecnologías fue fundamental), el proyecto del primer submarino brasileño de propulsión nuclear, postergado desde la década de 1970, tomó impulsó a través del Programa de Desarrollo de Submarinos (PROSUB) iniciado en septiembre de 2011 y la creación de la empresa estatal Amazônia Azul Tecnologias de Defesa (AMAZUL) en 2012, destinada al desarrollo de las tecnologías necesarias para las actividades de la Marina y el programa nuclear brasileño (Red de Seguridad y Defensa de América Latina - RESDAL, 2012). En agosto de ese año, y tras la aprobación del Senado, se dio inicio a la fase de diseño de las embarcaciones cuya culminación hacia 2016 debía dar paso a la construcción de la primera unidad y su puesta en servicio entre 2021 y 2025 bajo la denominación SN-BR. El programa se enmarcaba en una gran estrategia destinada a fortalecer las capacidades militares y la industria nacional de defensa del país a través del incremento en el gasto directo en armas y la transferencia tecnológica en cada acuerdo de compra. Entre los periodos 2001-2005 y 2006-2010 el volumen de importación directa en armamentos se había incrementado en un 436\% (Holtom et al., 2011; 2012). El fortalecimiento del poderío militar del país debía ser una señal de su creciente capacidad para influir en el

\footnotetext{
${ }^{7}$ El Libro Blanco de defensa de las Fuerzas Armadas del Brasil reconocían tres sectores estratégicos: nuclear, cibernético y espacial (MDB, 2012: 68-71).
} 
más amplio escenario de la política global y llevar a cabo un importante rol geoestratégico (Brands, 2010: 15), de ahí que el balance ambiguo del uso militar de la energía nuclear, pero con fines pacíficos, responda a objetivos fundamentalmente políticos. Haciendo énfasis en este aspecto, el profesor J. R. Martins Filho ha identificado el programa del submarino nuclear como un caso de "oportunismo tecnológico": las decisiones tomadas por un país en cuanto a tecnologías militares no parten de preferencias por un determinado armamento, sino de consideraciones estratégicas más amplias que encuentran en los armamentos un canal para su implementación (Martins Filho, 2011). El programa SNBR, con la nuclearización militar que implica, ha sido históricamente un instrumento de la gran estrategia de los sucesivos gobiernos. "Las élites políticas y militares no tienden a moldear sus estrategias en base a la tecnología militar, tienden en lugar de eso a ver la utilidad de las tecnologías a través de los lentes de sus estrategias actuales" (Lieber, 2005: 153). El mecanismo detrás de esta política permanente habría sido ya identificado: el programa de desarrollo del submarino nuclear respondería a un proceso de isomorfismo institucional (De Araújo de Assis, 2020), en el sentido de organizaciones (Estados) que compiten no sólo por recursos materiales sino por poder y legitimidad, así como aptitudes político-sociales y económicas (DiMaggio \& Powell, 1983: 150).

Además de considerar aspectos materiales del programa nuclear como actos efectivos que permiten inferir su orientación y propósitos, se encuentran también aspectos discursivos, de carácter valorativo, que tienen que ver con un difícil balance entre la adopción de una posición crítica frente a las desigualdades inherentes al régimen de no proliferación, y la defensa por una mayor apertura en los arreglos al interior del acuerdo. ¿Qué ideas están detrás de esta actitud ambigua del Brasil situada entre el compromiso y la denuncia al régimen de no proliferación y el uso militar de la energía atómica? Reivindicaciones asociadas a un auto-reconocimiento como una potencia global emergente (o la adopción de un rol en tal sentido), y la puesta en evidencia de la naturaleza instrumental de las instituciones internacionales (especialmente aquellas codificadas en instrumentos formales).

La literatura aborda este segundo conjunto de ideas tanto desde la perspectiva del actor internacional (el Estado-nación) como de la configuración del orden internacional. Dado el alcance de sus intereses en el más amplio escenario global, las grandes potencias tienen incentivos suficientes para la búsqueda de un mayor control en la gestión de los asuntos internacionales que aseguren un grado de predictibilidad en los resultados en función de sus objetivos políticos y económicos (Waltz, 1979: 199). En este sentido, el elemento material del poder nacional no sería suficiente para influir en los procesos políticos y sus resultados. Las instituciones internacionales - como un conjunto de normas y reglas que configuran la conducta y expectativas - son especialmente importantes para la gestión de la capacidad de influencia política de una gran potencia en la medida en que reflejan la jerarquía del poder internacional a través de ideas acordes con sus principales intereses y que contribuyen al logro de sus fines y objetivos (Mearsheimer 1994-1995: 13; Waltz, 2000: 26). Estos aspectos condensan algunos de los componentes principales del orden internacional: en un sentido general, una organización internacional que le otorga al orden establecido la forma de un arreglo colectivo (sobre una amplia aceptación que garantice su funcionamiento); en un sentido específico, la conformación de regímenes sectoriales que permiten a las grandes potencias regular áreas de especial interés (Alcalde, 2017: 1819). En Sudamérica, aunque difícilmente pueda hablarse de un orden hegemónico de parte del Brasil, proyectos como el IIRSA, en la parte funcional de la regionalización, o UNASUR, del lado de la coordinación política (a través de su Consejo de Defensa Sudamericano), fueron instancias regionales concebidas como parte importante en la 
estrategia de una gran potencia en ascenso orientada a la constitución de un bloque regional.

No es extraño entonces que Brasil asumiera una actitud crítica y reactiva frente a la asimetría de intereses que subyacen en el régimen de no proliferación nuclear desde sus orígenes. El 21 de marzo de 1977, el embajador de Brasil en Bonn, Alemania Occidental, reportaba al Ministerio de Relaciones Exteriores la información difundida en el diario Der Spiegel sobre las presiones ejercidas por la Unión Soviética, en colaboración con Estados Unidos, sobre el gobierno alemán para la revisión del acuerdo de cooperación nuclear con el Brasil y evitar así la transferencia tecnológica del enriquecimiento de uranio por el método de centrifugación (que finalmente quedaría fuera del acuerdo). El argumento: la supuesta responsabilidad global de las superpotencias (Wilson Center Digital Archive [WCDA], 1977). Lo cierto es que la cooperación a nivel político entre ambas superpotencias fue clave en la creación del régimen de no proliferación nuclear. En 1963, el embajador soviético en Polonia, Averky Aristov, informaba a su gobierno sobre el estado de las conversaciones con EE.UU. (aparentemente iniciadas en 1962) en torno al establecimiento de un futuro tratado de no proliferación nuclear, importante desde el punto de vista soviético para evitar que Alemania Occidental u otro miembro de la OTAN pudiera obtener o fabricar armas nucleares (WCDA, 1963). La visión de que el TNP representa una concepción asimétrica del mundo es una posición discrecional frente a una institucionalidad que codifica derechos extremadamente reducidos a unos pocos. ¿Qué puede decirse, entonces, de que Brasil asumiera hasta hoy la no proliferación como parte de su propia política nuclear? Es una actitud pragmática que ha equilibrado el rechazo y sujeción al régimen de no proliferación. La denuncia del TNP como ilegítimo ha permitido a los gobiernos del país desde la década de 1970 oponerse tanto a la posesión de arsenales nucleares de parte de unos pocos, como a los controles y la fiscalización sobre su propio programa atómico, promoviendo la crítica interna a la vez que instrumentalizando esa actitud en defensa del derecho al desarrollo tecnológico autónomo (Barletta, 1997: 19). Como parte de un compromiso calculado con el régimen de no proliferación, Brasil ha sometido su programa nuclear únicamente a las salvaguardias de la $\mathrm{ABACC}$, una organización binacional sobre la que mantiene un control más directo. La política exterior del país fue selectiva en la adopción de las normas y procedimientos del régimen de no proliferación que garantizarían su autonomía.

En el plano discursivo, Brasil pudo evitar las controversias que rodearon el desarrollo de otros programas nucleares a través de la auto-atribución de un compromiso con la paz y el diálogo internacionales que, paradójicamente, acompañaron al fortalecimiento de las capacidades militares del país hacia la primera década de los 2000. Durante la inauguración de la Unidad de Fabricación de Estructuras Metálicas, infraestructura de importancia para el PROSUB, la presidenta Rousseff afirmó que "el hecho de que Brasil sea un país pacífico, sin conflictos con sus vecinos, no es motivo para no tener una industria de defensa fuerte [...] La industria de defensa es una industria de paz, pero también, y sobre todo, es una industria de conocimiento" (Revista Naval, 2013). Pero las contradicciones evidentes en la actualidad entre esta retórica de paz y la realidad de un programa nuclear autónomo con fines militares, traen a la memoria el significado que tuvo para el Brasil la adhesión al TNP en 1997: según declarara entonces un alto funcionario del gobierno, "con su decisión de adherirse al Tratado, Brasil demuestra que quiere contribuir a la paz y que no tiene pretensiones militares en el área nuclear; que el país sólo quiere desarrollar proyectos nucleares pacíficos, como reactores de energía (...)" (La Nación, 1997). 
La ambigüedad de la política nuclear brasileña no se limita a su relación con las instituciones internacionales o a sus elementos discursivos. El actual grado de desarrollo de sus capacidades técnicas pone en cuestión el alcance del compromiso del país con la no proliferación —o mejor dicho, con la desnuclearización militar- y las tecnologías para su desarrollo. Por un lado, cuenta con capacidades técnicas necesarias para enriquecer uranio en dos instalaciones distintas: el Centro Experimental Aramar en São Paulo, que pertenece al Programa Nuclear de la Marina y está destinado a proveer de combustible al Programa de Submarinos Nucleares, y la planta de enriquecimiento de Resende, en Rio de Janeiro, en funcionamiento desde 2009, con capacidad para satisfacer las futuras necesidades de las centrales de energía Angra (1, 2 y 3) (Spanish China, 2012). Con esto, parte del uranio enriquecido habría de ser utilizado en áreas militares restringidas, excluidas de los mecanismos de control y fiscalización de la OIEA (Rühle, 2010; Stalcup, 2012). Por otro lado, la publicación de la tesis doctoral Simulação numérica de detonações termonucleares em meios Híbridos de fissão-fusão implodidos pela radiação del físico Dalton E. G. Barroso del Instituto de Ingeniería Militar del Ejército en 2009, confirmó que Brasil ya domina los conocimientos teóricos para producir un arma nuclear. El autor, que logró interpretar los modelos físicos y matemáticos de una cabeza nuclear desarrollada en los Estados Unidos, defendió su investigación afirmando que no era necesario construir un arma para saber cómo se fabrica (Arias, 2009; Quadros, 2009). Las preocupaciones en torno a la proliferación nuclear provienen, en cuanto a sus aspectos técnicos, del traslape en las tecnologías de doble uso. El desarrollo técnico asociado a un programa civil de energía nuclear (entre ellos, el enriquecimiento de uranio) puede ser reorientado a la fabricación de armas nucleares, lo que describe el llamado "dilema del doble uso" en el ámbito de la no proliferación (Miller \& Sagan, 2009: 13). En cuanto a Brasil, el desarrollo independiente de aplicaciones civiles y militares para la energía nuclear, las tecnologías de doble uso potencial y una actitud ambigua respecto al régimen de no proliferación, habían generado dudas comprensibles sobre su compromiso con el régimen de no proliferación y la desnuclearización regional (sobre este aspecto, ver Garay y Pérez, 2013).

Pero mientras no existan indicios inequívocos de que Brasil posee un programa de armas nucleares ${ }^{8}$, ésta debería ser una preocupación menor en el análisis de su orientación y consecuencias. Las aplicaciones militares de la energía nuclear sobre las que sí se tienen certezas - los generadores del programa PROSUB, especialmente - producirán efectos distintos al poder disuasivo de la Revolución Nuclear, pero igual de significativos sobre la seguridad internacional: entre otras cosas, porque la introducción de nuevas tecnologías militares puede exacerbar el dilema de seguridad, reavivando las dinámicas competitivas que subyacen en la región y a nivel hemisférico. Pocos días después de que Brasil retomara el proyecto de construcción de submarinos a propulsión nuclear en julio de 2011, el entonces ministro de defensa de Argentina, Arturo Puricelli, anunciaba que su país también buscaría desarrollar su propio submarino atómico (BBC Mundo, 2011; Clarín, 2011). Teniendo en cuenta estas reacciones tempranas - aunque descartadas de momento-, cabría preguntarse cuáles serían las consecuencias políticas de un nuevo actor nuclear en Sudamérica y cómo reaccionarían los países de la región y las grandes potencias frente a este escenario.

\footnotetext{
${ }^{8}$ Sobre las condiciones técnicas para la fabricación de la bomba atómica en Brasil, ver Sábato (1978). Aunque el informe de Jorge Sábato data de la década de 1970, los detalles técnicos descritos muestran aspectos básicos de lo que en adelante ha constituido del programa nuclear brasileño hasta la actualidad.
} 


\section{CONCLUSIONES}

¿Hacia dónde se dirige el programa nuclear del Brasil? No es una pregunta sobre metas y objetivos específicos, específicamente técnicos, sino sobre sus propósitos políticos y sus consecuencias en el mediano plazo. En este aspecto radica la primera diferencia importante entre las dos formas opuestas de entender su origen y desarrollo: la ausencia de un programa de armas nucleares puede inspirar cierta condescendencia en el análisis sobre sus objetivos políticos, pero, por otra parte, la ambiguiedad de las aplicaciones militares ya en curso deja espacio para el escepticismo respecto a un compromiso irrestricto con la no-militarización del poder atómico. Una perspectiva de poder, a diferencia de una interpretación más optimista al respecto, incidiría en esta cualidad, la ambigüedad, en lugar de sobrestimar la respetabilidad de un Estado según sus compromisos con la democracia, la institucionalidad internacional o la responsabilidad con los principios de la comunidad internacional.

El segundo aspecto que distingue a ambas interpretaciones, y que se desprende de lo anterior, es el peso otorgado al pragmatismo. Las interpretaciones optimistas se basan, generalmente, en las tesis liberales según las cuales los Estados guiarían su política exterior acorde con preceptos asociados a la superioridad moral de la democracia, las normas del derecho internacional público y el tribunal de la opinión pública. Pero desde una perspectiva opuesta, estas instancias están sujetas a la discrecionalidad de los Estados en función de una jerarquía que establece de antemano un marco de posibilidad y eficacia para sus acciones. En resumen, el pragmatismo en política exterior implica una cierta selectividad en el apego a los principios normativos de las relaciones internacionales. En lo que respecta a la política nuclear y el régimen de proliferación, su dimensión práctica más importante, la institucionalidad no puede sostenerse sobre esta clase de posturas: la restricción de ciertas acciones, y la prescripción de la conducta, dos formas en que las instituciones condicionan el comportamiento de los Estados, no pueden depender de un cumplimiento selectivo y calculado de la normatividad. Una lectura de la verdadera orientación y propósitos de un programa nuclear debería reconocer estos matices. ¿Pueden el gobierno y las Fuerzas Armadas del Brasil asegurar al resto de la comunidad internacional que los materiales nucleares de su programa serán utilizados con fines pacíficos? ¿Cómo encaja en esto el programa naval PROSUB/SN-BR? ¿Debería la noproliferación, como un principio asumido, referirse a un programa de armas nucleares o a un programa nuclear con fines militares?

Los dos puntos anteriores conducen a un tercer aspecto, más analítico que práctico. El programa nuclear del Brasil, con la ambigüedad y el pragmatismo que lo caracteriza, abre al menos dos nuevas vías de reflexión conceptual. Por una parte, la distinción entre lo que podría entenderse como un uso pacífico de la energía nuclear, esencialmente civil, en oposición al desarrollo de tecnologías militares a partir de este recurso, especialmente grandes arsenales. Por otra parte, la necesidad de repensar el propio concepto de noproliferación, que en un sentido restrictivo se refiere únicamente a la fabricación u obtención vetada de la bomba atómica, pero en un sentido amplio, a la fabricación u obtención de sistemas de armas basadas en tecnologías nucleares de doble uso. Finalmente, en el centro de éste y los otros aspectos, se ubican dos interpretaciones opuestas acerca de la orientación política y las implicaciones de un Brasil nuclear.

NOTA SOBRE EL AUTOR:

Juan Francisco Morales Giraldo. Maestro en Sociología en Estudios Políticos por la Universidad Nacional Mayor de San Marcos (UNMSM). Licenciado en Ciencia Política 
por la Pontificia Universidad Católica del Perú (PUCP). Es miembro investigador del Instituto de Estudios Políticos Andinos (IEPA) y del Grupo de Trabajo "Integración y Unidad Latinoamericana" del Consejo Latinoamericano de Ciencias Sociales (Clacso). Ha desempeñado labores de docencia en la UNMSM y en la PUCP. Correo electrónico: jfmoralesg.88@gmail.com

\section{REFERENCIAS}

Alcalde, Javier (2017), Las potencias del cambio. Rusia, India y China en la transformación del orden internacional, Lima: Instituto de Estudios Internacionales (IDEI-PUCP).

Amorim, Celso (2010), “A Política Externa Brasileira no governo do Presidente Lula (2003-2010) uma visão geral”, Revista Brasileira de Política Internacional, No. 53, pp. 214-240.

Amorim, Celso (2013), Los desafios del escenario estratégico del siglo XXI para América del Sur, Conferencia del Ministro de Estado de la Defensa, Celso Amorim, en el Ministerio de Defensa de Argentina, Buenos Aires, 13 de septiembre.

Arias, Juan (2009), "Revuelo nuclear en Brasil”, El País, 8 de septiembre.

Barletta, Michael (1997), Military Nuclear Program in Brazil, California: Center for International security and Arms Control (CISAC) - Stanford University.

Bandarra, Leonardo (2019), "Brazilian nuclear policy under Bolsonaro: no nuclear weapons but a nuclear submarine", TheBulletin.org, April 12: https://thebulletin.org/2019/04/brazilian-nuclear-policy-under-bolsonaro/

BBC Mundo (2011), “Sudamérica, a propulsión nuclear”, 4 de agosto.

Bompadre, Gerardo E. (2000), “Cooperación Nuclear Argentina-Brasil. Evolución y Perspectivas”, Relaciones Internacionales, Vol. 9, No. 18, pp. 53-62.

Brands, Hal (2010), Dilemmas of Brazilian Grand Strategy, Carlisle, Pennsylvania: Strategic Studies Institute (SSI) - US Army War College.

Carlson. John (2019), "Is the NPT Still Relevant? How to Progress the NPT's Disarmament Provisions", Journal for Peace and Nuclear Disarmament, Vol. 2, No. 1, pp. 97-113.

Clarín (2011), “Argentina fabrica submarino nuclear", 3 de agosto.

Dalton, Toby; Hoffman, Wyatt; Levite, Ariel E.; Bin, Lin; Perkovich, George \& Zhao, Tong (2017), Toward a Nuclear Firewall. Bridging the NPT's Three Pillars, Carnegie Endowment for International Peace.

Dantas, Vera (2017), "Entrevista: Almirante Bento Costa Lima Leite de Albuquerque Jr., Marinha do Brasil", Ministério da Defesa, Marinha do Brasil: https://www.marinha.mil.br/content/entrevista-almirante-bento-costa-lima-leite-dealbuquerque-jr

De Araújo de Assis, Jonathan (2020), "Normas, isomorfismo institucional e demanda militar: o projeto do submarino nuclear da Marinha do Brasil", Revista Neiba, Vol. 9, pp. 1-21. 
DiMaggio, Paul J. \& Powell, Walter W. (1983), "The Iron Cage Revisited: Institutional Isomorphism and Collective Rationality in Organizational Fields", American Sociological Review, Vol. 48, No. 2, pp. 147-160.

El Mundo (2011), "Rousseff dice que Brasil construirá submarinos para garantizar su soberanía", 18 de julio.

El Universal (2011), "Rousseff demanda ante la ONU destruir los arsenales nucleares", 22 de septiembre.

Feaver, Peter (2009). "What is grand strategy and why do we need it?", Foreign Policy, April 8: https://foreignpolicy.com/2009/04/08/what-is-grand-strategy-and-why-do-weneed-it/

Flemes, Daniel (2006), Brazil's Nuclear Policy. From Technological Dependence to Civil Nuclear Program, GIGA working papers, No. 23: http://www.gigahamburg.de/dl/download.php?d=/content/publikationen/pdf/wp23_flemes.pdf

Fondo Monetario Internacional [FMI] (2019), Report for Selected Country Groups and Subjects (PPP valuation of country GDP), World Economic Outlook Database.

Garay, Cristián y Pérez Luis V. (2013), "Brasil, el prestigio y el dilema del poder nuclear", Documento de Opinión, Instituto Español de Estudios Estratégicos.

Grabendorff, Wolf (1987), "La política nuclear y de no-proliferación de Brasil”, Estudios Internacionales, Vol. 20, No. 80, pp. 520-568.

Grieco, Joseph (1988), "Anarchy and the Limits of Cooperation: A Realist Critique of the Newest Liberal Institucionalism”, Internazional Organization, Vol. 2, No. 3, pp. 485507.

Habbeb, William M. (2000), "US-Egyptian Aid Negotiations in the 1980s and the 1990s", in Zartman, I. William \& Jeffrey, Z. Rubin (Eds.), Power and Negotiation, Ann Arbor: University of Michigan Press.

Holtom, Paul; Béraud-Sudreau, Lucie; Bromley, Mark; Wezeman, Pieter D. \& Wezeman, Siemon T. (2011), Trends in the International Arms Tranfers, 2010, SIPRI Fact Sheet, Estocolmo: Instituto de Investigación para la Paz Internacional de Estocolmo (SIPRI).

Holtom, Paul; Bromley, Mark; Wezeman, Pieter D. \& Wezeman, Siemon T. (2012). Trends in the International Arms Tranfers, 2011, SIPRI Fact Sheet, Estocolmo: Instituto de Investigación para la Paz Internacional de Estocolmo (SIPRI).

Holsti, Ole (1992), "Public Opinion on Foreign Policy: Challenges to the AlmondLippmann Consensus", International Studies Quarterly, Vol. 36, No. 4, pp. 439-466.

Jackson, Robert \& Sørensen, Georg (2007), Introduction to International Relations: Theories and Approaches, Tercera edición, New York: Oxford University Press.

Jervis, Robert (1986), “The Nulcear Revolution and the Common Defense", Political Science Quarterly, Vol. 101, No. 5, pp. 689-703.

Kahhat, Farid (2007), Tras la Guerra Fría. Mentalidad militar y políticas de seguridad en Sudamérica, Lima: Fondo Editorial del Congreso de la República.

Keohane, Robert (1993), Instituciones internacionales y poder estatal: Ensayo sobre la teoría de las Relaciones Internacionales, Buenos Aires: Grupo Editor Latinoamericano. 
Keohane, Robert O. (2009), "Una reconsideración del liberalismo internacional”, en Tamayo, Arturo B. (Comp.), Interdependencia, cooperación y globalismo, México D.F.: CIDE, pp. 45-88.

La Nación (1997), "Brasil se adhirió al Tratado de No Proliferación Nuclear”, 20 de junio.

La Nación (2010), "Lula: 'Son las armas nucleares y no los acuerdos con Irán lo que hace al mundo más peligroso", 28 de mayo.

La Voz del Mundo (2010), "Brasil no firmará el protocolo adicional de no proliferación", 29 de marzo.

Lieber, Keir A. (2005), War and the engineers, Londres: Cornell University Press.

Lomax, Lucius (2011), "What are the real reasons behind Brazil's interest in a nuclear powered submarine?", MercoPress, November http://en.mercopress.com/2011/11/26/what-are-the-real-reasons-behind-brazil-s-interestin-a-nuclear-powered-submarine

Martin, Félix (2003), "La hipótesis de la paz democrática y su análisis en el contexto suramericano”, Revista Quórum, Vol. 2, No. 4, pp. 91-103.

Martins Filho, Joao R. (2011), "O projeto do submarino nuclear brasileiro", Contexto Internacional, Vol. 33, No. 2, pp. 277-314.

Mauger, Philippe (2012), "Rejecting the Bomb: Conflicting Conclusions from Argentina and Brazil”, Américas: The John's Hopkins Journal of Latin America Studies, Vol. 3: http://www.americasjhu.org/p/current-edition.html

Mazui, Guilherme (2018), "Bolsonaro anuncia almirante Bento Costa Lima como futuro ministro de Minas e Energia", Globo.com, 30 de noviembre.

Mearsheimer, John J. (1994-1995), "The False Promise of International Institutions", International Security, Vol. 19, No. 3, pp. 5-49.

Mearsheimer, John J. (2001), The Tragedy of Great Power Politics, New York: W. W. Norton \& Company.

Miller, Steven E. \& Sagan, Scott D. (2009), "Nuclear power without nuclear proliferation?" Daedalus, Vol. 138, No. 4, pp. 7-18.

Ministerio de Defensa del Brasil (2008), Estrategia Nacional de Defensa. Paz y seguridad para Brasil (primera edición), Brasilia: Ministerio de Defensa, Gobierno Federal.

Ministerio de Defensa del Brasil (2012), Livro Branco de Defesa Nacional, Brasilia: Ministerio de Defensa, Gobierno Federal: http://www.defesa.gov.br/arquivos/2012/mes07/lbdn.pdf

Morales Giraldo, Juan F. (2018), "Las bases estructurales del liderazgo internacional en Sudamérica: análisis y medición de la influencia potencial y su distribución regional", Trabajo preparado para su presentación en la $8^{\circ}$ Conferencia Latinoamericana y Caribeña de Ciencias Sociales del el Consejo Latinoamericano de Ciencias Sociales (CLACSO). DOI: 10.13140/RG.2.2.15482.11202.

Morgenthau, Hans (1986), Política entre las Naciones. La lucha por el poder y la paz, Buenos Aires: Grupo Editor Latinoamericano.

Da Motta, Pedro y Ríos, Sandra P. (2007), O Regionalismo pós-liberal na América do Sul: origens, iniciativas e dilemas, Serie Comercio Internacional, No. 62, julio, Santiago de Chile: CEPAL. 
Nadal, Alejandro (1991), "La erosión del régimen de no proliferación de armas nucleares", Foro Internacional, Vol. 31, No. 4, pp. 546-573.

Nedal, Dani K. (s.d.), US Diplomatic Efforts Stalled Brazil's Nuclear Program in 1970, Wilson Center, NPIHP Research Updates: http://www.wilsoncenter.org/publication/usdiplomatic-efforts-stalled-brazils-nuclear-program-1970s

Oppenheimer, Andrés (2009), “AArmas Nucleares en América Latina?”, Informe21, 19 de octubre: http://informe21.com/blog/andres-oppenheimer/armas-nucleares-americalatina

Organización de Las Naciones Unidas (2010), "Brasil: armas nucleares no tienen utilidad", Centro de Noticias ONU, 10 de mayo: http://www.un.org/spanish/News/

Patti, Carlos (2010), "Brazil and the nuclear issues in the years of the Luiz Inácio Lula da Silva government (2003-2010)", Revista Brasileira de Política Internacional, Vol. 2, No. 53, pp. 178-197.

Patti, Carlo (s.d.), Origins and Evolution of the Brazilian Nuclear Program (1947-2011), Wilson Center, NPIHP Upearch http://www.wilsoncenter.org/publication/origins-and-evolution-the-brazilian-nuclearprogram-1947-2011

Quadros, Vasconcelos (2009), “A explosiva descoberta brasileira”, Jornal do Brasil, 5 de septiembre: $\quad$ http://www.jb.com.br/pais/noticias/2009/09/05/a-explosiva-descobertabrasileira/

Red de Seguridad y Defensa de América Latina (2012), Atlas Comparativo de la Defensa en América Latina y el Caribe - Edición 2012, Buenos Aires.

Revista Naval (2013), "Dilma Rousseff inaugura las instalaciones industriales para la producción de submarinos", 2 de marzo: http://www.revistanaval.com/noticia/20130302030942-dilma-rousseff-inaugura-ufem-prosub-itaguai/

Rühle, Hans (2010), "Nuclear Proliferation in Latin America: Is Brazil Developing the Bomb?" Spiegel Online, May 7: http://www.spiegel.de/international/world/nuclearproliferation-in-latin-america-is-brazil-developing-the-bomb-a-693336.html

Russell, Roberto \& Tokatlian, Juan G. (2003), "From Antagonistic Autonomy to Relational Autonomy: A Theoretical Reflection from the Southern Cone", Latin American Politics and Society, Vol. 45, No. 1, pp. 1-24.

Sábato, Jorge A. (1978), "El plan nuclear brasileño y la bomba atómica", Estudios Internacionales, Año. 11, No. 41, pp. 73-82.

Sagan, Scott D. (1996-1997), "Why do States Build Nuclear Weapons?: Three Models in Search of a Bomb", International Security, Vol. 21, No. 3, pp. 54-86.

Sagan, Scott; Waltz, Kenneth \& Betts, Richard (2007), “A Nuclear Iran: Promoting Stability or Coupling Disaster?”, Journal of International Affairs, Vol. 60, No. 2, pp. 135150 .

Sanahuja, José A. (2014), "Enfoques diferenciados y marcos comunes en el regionalismo latinoamericano: alcances y perspectivas de UNASUR y CELAC", Pensamiento Propio, No. 19, pp. 115-158.

Santos Vieira de Jesús, Diego (2011), "Desde Angra hacia Teherán: la política nuclear brasileña bajo la administración Lula", América Latina Hoy, Vol. 58, pp. 103-120. 
Schavelzon, Salvador (2017), "El fin de ciclo progresista sudamericano", Nueva Sociedad (en línea), febrero: https://www.nuso.org/articulo/el-fin-de-ciclo-progresistasudamericano/

Spanish China (2012), "Brasil concluye el primer módulo de su fábrica de enriquecimiento de uranio", 27 de octubre: http://spanish.china.org.cn/science/txt/201210/27/content_26921280.htm

Stalcup, Travis (2012), "What is Brazil up to with its Nuclear Policy?", Georgetown Journal of International Affairs, October 10 : http://journal.georgetown.edu/2012/10/10/what-is-brazil-up-to-with-its-nuclear-policyby-travis-stalcup/

Tussie, Diana \& Riggirozzi. Pia (Eds.) (2012), The Rise of Post-hegemonic Regionalism, Países Bajos: Springer.

Waisman, Valentina (2010), "Agentina y Brasil: percepciones y posturas actuales frente al régimen de no proliferación nuclear", Revista Política Hoje, Vol. 19, No. 2, pp. 488559

Waltz, Kenneth N. (1979), Theory of International Politics, Reading, Massachusetts: Addison-Wesley Publishing Company.

Waltz, Kenneth N. (2000), "Structural Realism after the Cold War", International Security, Vol. 25, No. 1, pp. 5-41.

Wilson Center Digital Archive (1963), Memorandum by Ambassador Aristov, History and Public Policy Program Digital Archive, Translation from the Russian by Douglas Selvage; Gomulka's marginalia, from the Polish. AAN, KC PZPR, sygn. 2639, 1 de abril, pp. 335-37: http://digitalarchive.wilsoncenter.org/document/111581

Wilson Center Digital Archive (1977), Brazilian Embassy Cable, Brazilian Ambassador to Bonn Reports on Soviet Pressure on West Germany. History and Public Policy Program Digital Archive, Centro de Pesquisa e Documentação de História Contemporânea do Brasil (CPDOC), Fundação Getúlio Vargas (FGV), Azeredo da Silveira Archive, AAS mre pn 1974.08 .15 pp. 589-591, Obtenido y traducido por la Fundação Getúlio 21 Vargas, de marzo: http://digitalarchive.wilsoncenter.org/document/115218

Windt, András (2017), "Success and Failures of the Non-Proliferation Treaty Demonstrated in History", ESARDA Bulletin, pp. 108121. http://inis.iaea.org/search/search.aspx?orig_q=RN:49062078

Zöpel, Christoph (2008), La integración sudamericana como requisito para la independencia, Nueva sociedad, No. 216, pp. 25-34. 